\title{
Calculation of the Gravitational Constant $G$ Using Electromagnetic Parameters
}

\author{
Jesús Sánchez \\ Independent Researcher, Bilbao, Spain \\ Email: jesus.sanchez.bilbao@gmail.com
}

How to cite this paper: Sánchez, J. (2017) Calculation of the Gravitational Constant $G$ Using Electromagnetic Parameters. Journal of High Energy Physics, Gravitation and Cosmology, 3, 87-95.

http://dx.doi.org/10.4236/jhepgc.2017.31012

Received: September 15, 2016 Accepted: December 25, 2016

Published: December 29, 2016

Copyright $\odot 2017$ by author and Scientific Research Publishing Inc. This work is licensed under the Creative Commons Attribution International License (CC BY 4.0).

http://creativecommons.org/licenses/by/4.0/

\section{Abstract}

In this paper, we will derive the following formula for the value of the gravitational constant $G: \quad G=\frac{\pi \alpha q^{2}}{m_{e}^{2} \varepsilon_{0}} \mathrm{e}^{-\frac{1}{\sqrt{2} \alpha}}=6.6202087 \times 10^{-11} \mathrm{~m}^{3} \cdot \mathrm{kg}^{-1} \cdot \mathrm{s}^{-2}$ (1). This equation has only $0.81 \%$ error compared to the common accepted value $6.67408 \times 10^{-11} \mathrm{~m}^{3} \cdot \mathrm{kg}^{-1} \cdot \mathrm{s}^{-2}$ [1]. The parameters in the equation are the following: $\alpha$ the fine structure constant, $q$ the elementary charge, $m_{e}$ the mass of the electron, $\varepsilon_{0}$ the permittivity of the free space, $e$ the exponential function and $\pi$ the relation between a circumference and its diameter. Values attached: $\alpha=\frac{q^{2}}{2 \varepsilon_{0} h c}=7.297152568 \times 10^{-3} \quad$ [2] ,

$q=1.60217662 \times 10^{-19} \mathrm{C}[1], m_{e}=9.10938356 \times 10^{-31} \mathrm{~kg}[1]$,

$\varepsilon_{0}=8.85418782 \times 10^{-12} \mathrm{~m}^{-3} \cdot \mathrm{kg}^{-1} \cdot \mathrm{s}^{4} \cdot \mathrm{A}^{2} \quad[1], \pi=3.14159265359$ [3], $\mathrm{e}=2.71828182845[4], h=6.62607004 \times 10^{-34} \mathrm{~m}^{2} \cdot \mathrm{kg} \cdot \mathrm{s}^{-1}$ (Planck constant) [1], $c=299792458 \mathrm{~m} \cdot \mathrm{s}^{-1}$ (speed of light) [1]. As it can be checked, all of them are electromagnetic or mathematical constants and properties of the electron. No constant related to gravity has been used to arrive to this value. This formula has only $0.81 \%$ error numerically and has the correct units. And it is the output of this paper. To get to this formula, we will consider space as being composed by the particles that occupy it. These particles include mass particles and force carriers (as photons). The non-uniform distribution of these particles creates distortions in space. These distortions create the warping of space, making gravity appear as an emergent phenomenon. Having in mind these considerations for a special case (an isolated electron emitting photons) we get to this formula that validates the assumptions.

\section{Keywords}

Quantum Gravity, Concept of Space, Gravitational Constant, Emergent Gravity, Size of a Photon 


\section{Introduction}

As commented, we will consider space as composed by the particles that occupy it. This means, vacuum space does not exist as itself, space only exists when a particle (in most of the cases, force carriers as photons) occupy it.

This, that could seem rather speculative has been proposed already in several papers by different authors [5] [6] [7].

When the number of particles increases in a region of space, the space itself increases in that region, making distances longer [5] in that region of space. When the number of particles decreases in the same region of space, the distances become shorter (the space reduces or shrinks) [5].

The difference of distributions of these particles in the different areas of space, provokes distortions, warping, that has the known effect, gravity. Remember, that particles in this context mean not only mass particles but also force carriers (mainly photons that are everywhere in space).

In two of the papers commented above [5] [6], it has been shown that gravity can be understood as an effect of new space emitted by the masses, that creates the distortions of space that lead to gravity. In this paper, going further, this new space is considered to be created just because the new particles (mainly force carriers as photons) occupy and create this new space.

A way to try to validate this view, is to find the relation between the distribution and size of particles in a region of space and the effects they provoke (gravity).

To do so, we will take a region of space with only one mass particle (an electron) emitting photons. The space in that region is occupied (and thus created by) only by these photons. We will make a calculation of the space occupied by these photons and the consequent effects created by this (gravity). This way we will be able to calculate $\mathrm{G}$ as if it were an unknown constant and compare its value with the already known value $\left(6.67408 \times 10^{-11} \mathrm{~m}^{3} \cdot \mathrm{kg}^{-1} \cdot \mathrm{s}^{-2}\right)[1]$.

\section{Procedure}

To be able to isolate $\mathrm{G}$ and calculate it, we will make the following study:

-We will consider an isolated electron emitting photons.

-We will consider that the vacuum space surrounding this electron is only composed/occupied by these photons.

Anyhow, we will see that with this consideration we will get a very approximate calculation of $G$ (with only $0.81 \%$ error) (27).

\section{Electron as an Elementary Particle}

The calculations in this paper will be done always using electrons and their emitted photons.

The reason for using electrons is important. They are the most elementary particles that has a charge (the elementary charge in fact). This means:

-The mass of the electron can be considered as the total energy of the photons emitted by it (the total electrostatic energy created by them) [8]. This is not exact [9] but is a good approximation. This is not true for a proton for example. 
-The classical radius of an electron can be considered as the radius of a mass that is having the energy of all the photons emitted by it [8]. Again, not true for a proton for example.

\section{Radius of the Electron}

We will use the concept of classical radius of an electron $r_{e}$. This concept is well defined and described in the literature [8]. Its value [1] is:

$$
r_{e}=\frac{1}{4 \pi \varepsilon_{0}} \frac{q^{2}}{m_{e} c^{2}}=2.8179403267 \times 10^{-15} \mathrm{~m}
$$

\section{Size of a Photon}

In this study, we will consider a single photon as an elementary sine wave [10]. In a sine wave when the wavelength is $2 \pi$ the peak value is 1 [10], so the peak value in an elementary sine wave with arbitrary wavelength will be $\lambda / 2 \pi \quad[10]$.

$$
\text { peakvalue }=\frac{\lambda}{2 \pi}
$$

The root mean square (rms) value in a sine wave is the peak value divided by $\sqrt{2}$ [10] so:

$$
\text { rmsvalue }=\frac{\text { peakvalue }}{\sqrt{2}}=\frac{\lambda}{2 \pi \sqrt{2}}
$$

From now on, when we want to represent the rms value of an elementary sine wave with a specific wavelength $\lambda$ we will call it as $\lambda_{r m s}$ :

$$
\lambda_{\text {rms }}=\frac{\lambda}{2 \pi \sqrt{2}}
$$

As we will see later, the calculations will lead us to consider the size of the photon (or more specific, its radius) as the rms value of the wave of the photon according Equation (5).

$$
r_{f}=\lambda_{r m s}=\frac{\lambda}{2 \pi \sqrt{2}}
$$

This means, the total front surface of a photon will be $\pi r_{f}^{2}$. And the linear dimension of the photon, perpendicular to its movement direction will the diameter of this surface. This is, two times the rms value (value from $r m s$ to $r m s$ ):

$$
\text { Size of a photon perpendicular to its direction }=2 r_{f}=2 \lambda_{r m s}=\frac{2 \lambda}{2 \pi \sqrt{2}}=\frac{\lambda}{\pi \sqrt{2}}
$$

The size of a photon (or even if it has a size) has always been a controversial study. Anyhow, all the studies [11] [12] [13] conclude that if this size exists, it is somehow related to its wavelength, as we have considered before. In easy words, as commented in [14], if I try to shoot a photon through a conducting tube much smaller than a wavelength, it does not go through.

Later calculations will confirm that the consideration of the rms value matches with the expected results. 


\section{Increment of Space Created by an Electron}

As commented in chapter 1, gravity is considered as an emergent phenomenon (a side effect) of space being composed by particles (including force carriers as photons). This means, when a particle emits other particles to space, it is creating new space. And this new space is the one that is warping space, provoking gravity.

To be able to make a relation between space created by gravity and space created by new particles (in this case the photons emitted by an electron), we need to calculate the first one.

This will be the only time in the paper where we use a formula related to gravity.

We consider Schwarzschild equation [15]. This equation calculates the deformation of space in the different directions caused by a mass. It matches with our situation (the space surrounded by an isolated electron).

$$
\mathrm{d} s^{2}=-\left(1-\frac{2 G m}{c^{2} r}\right) \mathrm{d} t^{2}+\left(\frac{1}{1-\frac{2 G m}{c^{2} r}}\right) \mathrm{d} r^{2}+r^{2} \sin ^{2} \theta \mathrm{d} \vartheta^{2}+r^{2} \mathrm{~d} \theta^{2}
$$

In an instant of time and in radial direction we have:

$$
\mathrm{d} s^{2}=\left(\frac{1}{1-\frac{2 G m}{c^{2} r}}\right) \mathrm{d} r^{2} \frac{\mathrm{d} s}{\mathrm{~d} r}=\sqrt{\frac{1}{1-\frac{2 G m}{c^{2} r}}} \approx 1+\frac{G m}{c^{2} r}
$$

The first element 1 represents the no distortion (Euclidean space). So if we take the geometrical line that keep the same $r$ (the circumference), we can calculate the space incremented space in that line due to gravity as:

$$
\Delta \text { circumference }=2 \pi r\left(1+\frac{G m}{c^{2} r}\right)-2 \pi r=\frac{2 \pi G m}{c^{2}}
$$

This is the increment in of space in the circumferential direction. If we want to convert to the equivalent increment of space in radial direction we divide by $2 \pi$.

$$
\Delta \text { radius }=\frac{G m}{c^{2}}
$$

And we see that this increment is kept constant whatever the radius. This is logical, as the space created by the particle is the same at a precise moment of time and it is transferred outwards. Its effects reduce with distance as this new space is dispersed more, the bigger the radius. But the total increment of space of a total shell is the same independently of the radius.

At this moment, we will make a little counter-intuitive trick. We will consider this increment of space also as a wave and we will apply the same considerations of chapter 5.

This means, we calculate the effective increment of space as two times the rms value (rms to rms value) of the $\Delta$ radius:

$$
\Delta \text { incrementofspace }=\frac{2 G m}{\sqrt{2} 2 \pi c^{2}}=\frac{G m}{\sqrt{2} \pi c^{2}}
$$

With the same considerations as in chapter 5 . We will see that these considerations 
work, as we will be able to get to an equation of $G(1)$ with only $0.81 \%$ error (27).

\section{Wavelength of the Photons Emitted by an Electron}

For this paper we have to consider a mean value of the wavelength of the photons emitted by an electron. Be careful here, the electron emits two type of photons: real photons and virtual photons.

The real photons and its wavelength depends on its level of energy in the atom and the necessary energy to emit them or change its level, that as we know is quantized.

But the virtual photons are a complete unknown black box. It is known that if we consider them as having all the possible wavelengths, this matches with some effects [16] [17]. But the mean value of this wavelength is unknown.

For this paper we will consider mean value of the wavelength as the Compton wavelength of the particle that emits them. In the case of the electron this leads to:

$$
\lambda_{e}=\frac{h}{m_{e} c}=2.4263102 \times 10^{-12} \mathrm{~m}
$$

This consideration will be very probably only valid in elementary particles. As in this case we will use always use electrons (elementary particles) throughout the report, we can avoid the discussion.

If we calculate now the double of the root mean square value commented in chapter 5 would be:

$$
2 \lambda_{\text {rms }}=\frac{2 h}{\sqrt{2} 2 \pi m_{e} c}=\frac{h}{\sqrt{2} \pi m_{e} c}=5.4611167 \times 10^{-12} \mathrm{~m}
$$

This would be the diameter occupied by the photon (in perpendicular direction of propagation).

\section{Definition of the Magnitude $y(r)$}

We will define the following magnitude:

$$
y(r)=\frac{\Delta S(r)}{S(r)}=\frac{\Delta S(r)}{4 \pi r^{2}}
$$

In a given shell of theoretical surface $S$ and radius $r, \Delta S$ is defined as the increment of surface created by the emitted photons.

This means, $y$ is the ratio, in a given shell of radius $r$, between the increment of surface created by the photons and the theoretical surface if these photons did not exist.

\section{Equation}

Now, we have all the elements needed to perform the calculation. We will create an equation, defining first the left hand side and the right hand side separately. Afterwards, we will make them equal.

To get to the equation, we will calculate the effects on space provoked by the photons emitted by the electron, in two different ways. We will start talking about a general position $r$, but finally we will apply to the radius $r_{e}$ (radius of the electron). This way, we will arrive to an equation where all the data are known (an "over defined" equation). 
We will isolate $G$ and calculate it. We will discover that the calculated value of $G$ will have only $0.81 \%$ error compared to real value of $G(27)$.

\section{Calculation of the Left Hand Side}

We know that the magnitude $y(r)$ is depending on the surface itself. So, if we want to calculate the total creation of surface created by the photons (in a shell of radius $r$ ), we have to integrate the variation of this quantity divided by the quantity itself from infinitum to the radius $r$.

In another way, the total increment of this magnitude depends on the size of the magnitude itself. This can be written as:

$$
\int_{\infty}^{r} \frac{\mathrm{d} y}{y(r)}
$$

As can be checked, it will not be possible to solve all the definite integrals (with zeros or infinitein their limits) avoiding to have infinite result in return. This is because Equation (16) and Equation (18) in chapter 11 are mutually excluding. If one is defined to be convergent, the other one will be divergent and vice versa.

The way we will use to avoid this infinite result is the following. We will solve all the integrals as indefinite and will include the integration constant in the result. This way, the result is valid, as the integration constant will be calculated for whatever values of the variable we want to use.

$$
\int \frac{\mathrm{d} y}{y(r)}=\ln y(r)+C_{1}=\ln \left(\frac{\Delta S(r)}{4 \pi r^{2}}\right)+C_{1}
$$

Being $C_{1}$ the integration constant

\section{Right Hand Side of the Equation}

At each shell of $\mathrm{d} r$, the new space (in ratio) created by the photons will correspond to the size of the photon ( $2 \lambda_{r m s}$ of the photon in diameter) (7), divided by the unit of space at that shell in diameter $(2 r)$ multiplied by the advance unit of integral $(\mathrm{d} r)$ divided by the unit of space at that shell $(r)$.

So the total space created by the photons will correspond to:

$$
\int_{\infty}^{r} \frac{2 \lambda_{r m s}}{2 r} \frac{\mathrm{d} r}{r}
$$

As commented before, to avoid infinite results we will solve as indefinite integral with an integration constant.

$$
\int \frac{\lambda_{r m s}}{r} \frac{\mathrm{d} r}{r}=\int \frac{\lambda \mathrm{d} r}{\sqrt{2} 2 \pi r^{2}}=\frac{-\lambda}{\sqrt{2} 2 \pi r}+C_{2}
$$

Being $C_{2}$ the integration constant.

\section{Joining Both Hands of the Equation}

If we join the left hand and the right side of the equation we have:

$$
\ln \left(\frac{\Delta S(r)}{4 \pi r^{2}}\right)+C_{1}=\frac{-\lambda}{\sqrt{2} 2 \pi r}+C_{2}
$$


As this Equation (20) is valid for whatever radius, we will calculate the equation for a specific radius in order to create an "over defined" equation. This way, we will be able to isolate $G$ as if it were an unknown variable.

To create this "over defined" equation, we will calculate the equation for a specific radius, a radius that we know and we have specific data for it. This radius will be the radius of the electron $r=r_{e}$. Also, please take into account that the photons considered in this case are emitted by an electron so in this case $\lambda=\lambda_{e}(13)$ :

$$
\ln \left(\frac{\Delta S\left(r_{e}\right)}{4 \pi r_{e}^{2}}\right)+C_{1}=\frac{-\lambda_{e}}{\sqrt{2} 2 \pi r_{e}}+C_{2}
$$

Apart form $C_{1}$ and $C_{2}$, the only unknown variable is $\Delta S\left(r_{e}\right)$. We will calculate this value in the following chapter to be able to go on with the equation.

\section{Calculation of $\Delta S\left(r_{e}\right)$}

At the radius $r_{e}$, the $\Delta S$ is the multiplication of the created space in radial direction $\frac{G m}{\sqrt{2} \pi c^{2}}$ (12) multiplied by the height of the photon (we can consider it in axial or tangential direction) that is $\frac{h}{\sqrt{2} \pi m_{e} c}(14)$.

$$
\Delta S\left(r_{e}\right)=\frac{G m_{e}}{\sqrt{2} \pi c^{2}} \frac{h}{\sqrt{2} \pi m_{e} c}=\frac{G h}{2 \pi^{2} c^{3}}
$$

\section{Calculating the Equation}

If we recover the Equation (21):

$$
\ln \left(\frac{\Delta S\left(r_{e}\right)}{4 \pi r_{e}^{2}}\right)+C_{1}=\frac{-\lambda_{e}}{\sqrt{2} 2 \pi r_{e}}+C_{2}
$$

We will consider the two integration constants equal to zero. This will be the same as choosing the start and end of the corresponding definite integral in a way that we get no infinite result. We will see later that this consideration works, as we will get an error for $G$ of $0.81 \%$ with this assumption.

$$
\ln \left(\frac{\Delta S\left(r_{e}\right)}{4 \pi r_{e}^{2}}\right)=\frac{-\lambda_{e}}{\sqrt{2} 2 \pi r_{e}}
$$

Using (22):

$$
\begin{array}{r}
\ln \left(\frac{G h}{4 \pi r^{2} c^{3}}\right)=\frac{-\lambda_{e}}{\sqrt{2} 2 \pi r_{e}} \\
\ln \left(\frac{G h}{8 \pi^{3} c^{3} r_{e}^{2}}\right)=\frac{-\lambda_{e}}{\sqrt{2} 2 \pi r_{e}}
\end{array}
$$

If we apply the exponential to both sides:

$$
\frac{G h}{8 \pi^{3} c^{3} r_{e}^{2}}=\mathrm{e}^{\frac{-\lambda_{e}}{\sqrt{2} 2 \pi r_{e}}}
$$


If we substitute $\lambda_{e}$ (13) and $r_{e}$ (2) we get:

$$
\frac{G h}{8 \pi^{3} c^{3}\left(\frac{q^{2}}{4 \pi \varepsilon_{0} m_{e} c^{2}}\right)^{2}}=\mathrm{e}^{\frac{\frac{-h}{m_{e} c}}{\sqrt{2} 2 \pi \frac{q^{2}}{4 \pi \varepsilon_{0} m_{e} c^{2}}}}
$$

If we isolate $G$ and use the definition of fine structure constant $\alpha=\frac{q^{2}}{2 \varepsilon_{0} h c}$ [2]:

$$
G=\frac{\pi \alpha q^{2}}{m_{e}^{2} \varepsilon_{0}} \mathrm{e}^{-\frac{1}{\sqrt{2} \alpha}}=6.6202087 \times 10^{-11} \mathrm{~m}^{3} \cdot \mathrm{kg}^{-1} \cdot \mathrm{s}^{-2}
$$

If we compare with the commonly accepted value of $6.67408 \times 10^{-11} \mathrm{~m}^{3} \cdot \mathrm{kg}^{-1} \cdot \mathrm{s}^{-2}$ [1]:

$$
\text { error }=\frac{6.67408 \times 10^{-11}-6.6202087 \times 10^{-11}}{6.67408 \times 10^{-11}} \times 100=0.81 \%
$$

As we have checked, all the assumptions considered (including eliminating the integration constants) have given a calculation of $G$ with only a $0.81 \%$ error!

\section{Conclusions}

We have proposed a theory that considers that space is composed by the particles (including mass particles and force carriers) that occupy it. Gravitation effects are nothing but a result of the composition of space and its irregularities causing it to warp in the different areas.

Even with the not intuitive assumptions commented in chapter 1 and the assumptions considered to perform the calculations, the result is surprising anyhow. Not only to get a result of the order of magnitude needed but to get only an error of $0.81 \%$ is really surprising.

According Equation (1):

$$
G=6.6202087 \times 10^{-11} \mathrm{~m}^{3} \cdot \mathrm{kg}^{-1} \cdot \mathrm{s}^{-2}
$$

While the known and accepted value is:

$$
G=6.67408 \times 10^{-11} \mathrm{~m}^{3} \cdot \mathrm{kg}^{-1} \cdot \mathrm{s}^{-2}
$$

This means an error of only $0.81 \%$ as can be checked in Equation (27).

In whatever theory, to arrive to a calculation of a constant of the order of magnitude of $10^{-11}$ with the correct measurement units and $0.81 \%$ error in the representative digits, should make us at least think seriously that this theory could at least go in the good direction.

The idea of space being composed or at least affected by the particles that occupy it (not only masses but whatever particles that occupy it) is a theory that has been proposed by several scientists in different papers as [5] [6] [7].

The added value of this paper is that the theory leads to a calculation of $G$ using only electromagnetic parameters that is a way of validating or at least giving some push to the theory. 


\section{Acknowledgements}

To my family and friends.

\section{References}

[1] Mohr, P.J., Taylor, B.N. and Newell, D.B. (2015) The 2014 CODATA Recommended Values of the Fundamental Physical Constants (Web Version 7.0). This database was developed by J. Baker, M. Douma, and S. Kotochigova. http://physics.nist.gov/constants

[2] Bouchendira, R., Cladé, P., Guellati-Khélifa, S., Nez, F. and Biraben, F. (2010) New Determination of the Fine-Structure Constant and Test of the Quantum Electrodynamics. Physical Review Letters, 106, 080801. arXiv:1012.3627Bibcode:2011PhRvL.106h0801B.

https://arxiv.org/abs/1012.3627 https://doi.org/10.1103/PhysRevLett.106.080801

[3] Arndt, J. and Haenel, C. (2006) Pi Unleashed. Springer-Verlag, New York. English Translation by Catriona and David Lischka.

[4] O'Connor, J.J. and Robertson, E.F. The Number e. MacTutor History of Mathematics.

[5] http://arxiv.org/abs/1105.2705

[6] http://vixra.org/abs/1509.0221

[7] http://www.scirp.org/Journal/PaperInformation.aspx?PaperID=67955

[8] Griffiths, D.J. (1995) Introduction to Quantum Mechanics, Prentice-Hall, Upper Saddle River, 155.

[9] Thomson, J.J. (1881) On the Electric and Magnetic Effects produced by the Motion of Electrified Bodies. Philosophical Magazine, 11, 229-249. https://doi.org/10.1080/14786448108627008

[10] Daintith, J. (2009) A Dictionary of Physics. 6th Edition, Oxford University Press, Oxford, UK.

[11] http://rcwww.kek.jp/research/shield/photon_r.pdf

[12] http://www.sciencedirect.com/science/article/pii/S0022407303001146

[13] https://briankoberlein.com/2015/04/14/thats-about-the-size-of-it/

[14] http://www.scienceforums.net/topic/27337-does-a-photon-have-physical-volume-or-geome trical-size/

[15] http://gfm.cii.fc.ul.pt/events/lecture_series/general_relativity/gfm-general_relativity-lecture $\underline{4 . p d f}$

[16] Shu, F.H. The Physical Universe. And Introduction to Astronomy, 108. https://books.google.es/books?id=v_6PbAfapSAC\&pg=PA108\&lpg=PA108\&dq=range+wav elength+virtual+photon\&source=bl\&ots=oqrksX7gjD\&sig=9nd3Cr5gK7MWDVuYGnLxL 1jZ2Ek\&hl=es\&sa $=$ X\&ved=0ahUKEwil29iSsYXPAhVBnBQKHcuiCkcQ6AEIMTAC\#v=on epage\&q=range $\% 20$ wavelength $\% 20$ virtual\%20photon\&f=false

[17] http://electron6.phys.utk.edu/phys250/modules/module\%206/standard_model.htm 
Submit or recommend next manuscript to SCIRP and we will provide best service for you:

Accepting pre-submission inquiries through Email, Facebook, LinkedIn, Twitter, etc. A wide selection of journals (inclusive of 9 subjects, more than 200 journals) Providing 24-hour high-quality service

User-friendly online submission system

Fair and swift peer-review system

Efficient typesetting and proofreading procedure

Display of the result of downloads and visits, as well as the number of cited articles Maximum dissemination of your research work

Submit your manuscript at: http://papersubmission.scirp.org/

Or contact jhepgc@scirp.org 